ELECTRONIC RESEARCH ANNOUNCEMENTS OF THE AMERICAN MATHEMATICAL SOCIETY

Volume 12, Pages 121-128 (August 18, 2006)

S $1079-6762(06) 00168-5$

\title{
ON THE DISTRIBUTION OF THE ORDER OVER RESIDUE CLASSES
}

\author{
PIETER MOREE
}

(Communicated by Brian Conrey)

\begin{abstract}
For a fixed rational number $g \notin\{-1,0,1\}$ and integers $a$ and $d$ we consider the set $N_{g}(a, d)$ of primes $p$ such that the order of $g$ modulo $p$ is congruent to $a(\bmod d)$. Under the Generalized Riemann Hypothesis $(\mathrm{GRH})$, it can be shown that the set $N_{g}(a, d)$ has a natural density $\delta_{g}(a, d)$. Arithmetical properties of $\delta_{g}(a, d)$ are described, and $\delta_{g}(a, d)$ is compared with $\delta(a, d)$ : the average density of elements in a field of prime characteristic having order congruent to $a(\bmod d)$. It transpires that $\delta_{g}(a, d)$ has a strong tendency to be equal to $\delta(a, d)$, or at least to be close to it.
\end{abstract}

\section{INTRODUCTION}

Let $g \notin\{-1,0,1\}$ be a rational number. For a rational number $u$, let $\nu_{p}(u)$ denote the exponent of $p$ in the canonical factorisation of $u$ (throughout, the letter $p$ will be used to indicate prime numbers). If $\nu_{p}(g)=0$, then there exists the least positive integer $k$ such that $g^{k} \equiv 1(\bmod p)$. We put $\operatorname{ord}_{p}(g)=k$. The number $k$ is the (residual) order of $g(\bmod p)$. We let $N_{g}\left(a_{1}, d_{1} ; a_{2}, d_{2}\right)$ be the set of primes $p$ with $\nu_{p}(g)=0, p \equiv a_{1}\left(\bmod d_{1}\right)$ and $\operatorname{ord}_{p}(g) \equiv a_{2}\left(\bmod d_{2}\right)$. By $N_{g}\left(a_{1}, d_{1} ; a_{2}, d_{2}\right)(x)$ we denote the number of primes $p \leq x$ in $N_{g}\left(a_{1}, d_{1} ; a_{2}, d_{2}\right)$. For convenience, $N_{g}(0,1 ; a, d)(x)$ is written as $N_{g}(a, d)(x)$. By GRH we denote the Generalized Riemann Hypothesis. By $(a, b)$ and $[a, b]$ we denote the greatest common divisor and the least common multiple of $a$ and $b$, respectively.

Theorem 1 ([M-2]). (GRH). The density $\delta_{g}\left(a_{1}, d_{1} ; a_{2}, d_{2}\right)$ of the set of primes $N_{g}\left(a_{1}, d_{1} ; a_{2}, d_{2}\right)$ exists. Moreover,

$$
N_{g}\left(a_{1}, d_{1} ; a_{2}, d_{2}\right)(x)=\delta_{g}\left(a_{1}, d_{1} ; a_{2}, d_{2}\right) \frac{x}{\log x}+O_{g, d}\left(\frac{x}{\log ^{3 / 2} x}\right) .
$$

Our primary interest is in $\delta_{g}(a, d)=\delta_{g}(0,1 ; a, d)$, but in studying this quantity it turns out to be fruitful to consider $N_{g}\left(a_{1}, d_{1} ; a_{2}, d_{2}\right)(x)$. Theorem [3, for example, is obtained from Theorem 4 . By $K_{s, r}$ (with $r \mid s$ ) we denote the number field $\mathbb{Q}\left(\zeta_{s}, g^{1 / r}\right)$, where $\zeta_{s}=\exp (2 \pi i / s)$. The density $\delta_{g}\left(a_{1}, d_{1} ; a_{2}, d_{2}\right)$ can be

Received by the editors February 5, 2006 and, in revised form, June 20, 2006.

2000 Mathematics Subject Classification. Primary 11N37, 11R45; Secondary 11N69.

(C)2006 American Mathematical Society 
expressed in terms of the degrees $\left[K_{s, r}: \mathbb{Q}\right]$ and certain intersection coefficients (with $(b, f)=1$ ),

$$
c_{g}(b, f, v)= \begin{cases}1 & \text { if }\left.\sigma_{b}\right|_{\mathbb{Q}\left(\zeta_{f}\right) \cap K_{v, v}}=\text { identity; } \\ 0 & \text { otherwise, }\end{cases}
$$

where $\sigma_{b}$ is the automorphism of $\mathbb{Q}\left(\zeta_{f}\right)$ that sends $\zeta_{f}$ to $\zeta_{f}^{b}$.

Theorem 2 ( $\mathrm{M}-2])$. (GRH). We have

$$
\delta_{g}(a, d)=\sum_{\substack{t=1 \\(1+t a, d)=1}}^{\infty} \sum_{\substack{n=1 \\(n, d) \mid a}}^{\infty} \frac{\mu(n) c_{g}(1+t a, d t, n t)}{\left[K_{[d, n] t, n t}: \mathbb{Q}\right]} .
$$

In the proofs of various results below, the determination of the intersection coefficients $c_{g}(b, f, v)$ plays an important rôle. Obviously $\mathbb{Q}\left(\zeta_{f}\right) \cap K_{v, v}$ is a subfield of the maximal abelian subfield, $K_{v, v}^{\mathrm{ab}}$, of $K_{v, v}$. It turns out (see [M-3]) that $K_{v, v}^{\mathrm{ab}}$ is of the form $\mathbb{Q}\left(\zeta_{v}, \sqrt{\gamma}\right)$ or of the form $\mathbb{Q}\left(\zeta_{v}, \zeta_{2 v} \sqrt{\gamma}\right)$ for some integer $\gamma$ that can be explicitly given, where the latter case does not arise if $g>0$. Of course, the action of $\sigma_{b}$ on $\mathbb{Q}\left(\zeta_{f}\right) \cap K_{v, v}=\mathbb{Q}\left(\zeta_{f}\right) \cap K_{v, v}^{\mathrm{ab}}$, which equals $\mathbb{Q}\left(\zeta_{f}\right) \cap \mathbb{Q}\left(\zeta_{v}, \sqrt{\gamma}\right)$ or $\mathbb{Q}\left(\zeta_{f}\right) \cap \mathbb{Q}\left(\zeta_{v}, \zeta_{2 v} \sqrt{\gamma}\right)$, is easily determined.

The distribution of the order over congruence classes in case $d \nmid a$ seems to have been first studied by Chinen and Murata [CM] for $d=4$. In case $d \mid a$ the problem is much easier and unconditional results have been obtained; see [M-0, W-1, W-2]. In this case the density is always a rational number. Chinen and Murata restricted themselves to the case where $g$ is positive and not a power of an integer. In their method, $\delta_{g}(a, 4)$ (for $a$ odd) is initially expressed as the sum of two fourfold sums. On making everything explicit, they obtained a long formula (distinguishing six cases) for $\delta_{g}(a, 4)$, which was subsequently simplified by Zagier [Z]. The author expressed $\delta_{g}(a, 4)$ as a single sum (see Theorem 4 ), which on evaluation gives a compact formula for $\delta_{g}(a, 4)$ similar to Zagier's. In this formula an Euler product $A_{\psi}$ appears. We put, for any Dirichlet character $\chi$,

$$
A_{\chi}=\prod_{\substack{p \\ \chi(p) \neq 0}}\left(1+\frac{[\chi(p)-1] p}{\left[p^{2}-\chi(p)\right](p-1)}\right) .
$$

The constants $A_{\chi}$ turn out to be rather basic in this setting (cf. Theorem 11). A table of numerical values of $A_{\chi}$, with $\chi$ a Dirichlet character of modulus $\leq 12$, is given in [M-0]. We let $\mathcal{G}$ be the set of rational integers that cannot be written as $-g_{0}^{h}$ or $g_{0}^{h}$ with $h>1$ an integer and $g_{0}$ a rational number. Note that almost all integers $g$ are elements of $\mathcal{G}$.

Theorem 3 ( $[\mathrm{M}-1])$. (GRH). Let $D(g)$ denote the discriminant of the field $\mathbb{Q}(\sqrt{g})$. Let $g \in \mathcal{G}$. Then $\delta_{g}( \pm 1,4)=1 / 6$ unless $D(g)$ is divisible by 8 and has no prime divisor congruent to $1(\bmod 4)$, in which case we have

$$
\delta_{g}( \pm 1,4)= \begin{cases}\frac{1}{6} \mp \operatorname{sgn}(g) \frac{A_{\psi}}{8} \prod_{p \mid \frac{D(g)}{8}} \frac{2 p}{p^{3}-p^{2}-p-1} & \text { if } D(g) \neq \pm 8 ; \\ \frac{7}{48} \mp \operatorname{sgn}(g) \frac{A_{\psi}}{8} & \text { if } D(g)= \pm 8,\end{cases}
$$

where $\psi$ denotes the non-trivial character modulo 4.

Remark 1. We have, on invoking Theorem 12, $A_{\psi}=0.643650679662525 \cdots$. 
Remark 2. Let $\epsilon_{1}(n)=1$ if $8 \mid n$ and 0 otherwise. Then we can write, using, e.g., Theorem 2 of Moree [M-0], for $2 \nmid a$ :

$$
\delta_{g}(a, 4)=\frac{\delta_{g}(1,2)}{2}+\epsilon_{1}(D(g)) \operatorname{sgn}(g) A_{\psi} \frac{(-1)^{\frac{a+1}{2}}}{8} \prod_{p \mid \frac{D(g)}{8}} \frac{(1-\psi(p)) p}{p^{3}-p^{2}-p-1} .
$$

An explicit expression for $\delta_{g}\left(a, q^{s}\right)$ (in terms of $A_{\chi}$ 's) with $q$ a prime and $g \in \mathcal{G}$ is obtained in [M-2, but is omitted here for reasons of space.

Theorem 3 can be obtained from the following result (with $s=2$ ):

Theorem 4 ([M-1]). (GRH). We have, for a odd and $s \geq 1$,

$$
N_{g}\left(1,2^{s} ; a, 4\right)(x)=\frac{\delta_{g}\left(1,2^{s} ; 1,2\right)}{2} \frac{x}{\log x}+O_{g}\left(\frac{x}{\log ^{3 / 2} x}\right),
$$

and $N_{g}(3,4 ; a, 4)(x)=\#\left\{p \leq x: p \equiv 3(\bmod 4),\left(\frac{g}{p}\right)=1\right\} / 2$

$$
+(-1)^{\frac{a-1}{2}} \frac{\Delta_{g}}{4} \frac{x}{\log x}+O_{g}\left(\frac{x}{\log ^{3 / 2} x}\right),
$$

where

$$
\Delta_{g}=\sum_{\substack{\sqrt{-2} \in K_{2 v}, 2 v \\ 2 \nmid v}} \frac{h_{\psi}(v)}{\left[K_{2 v, 2 v}: \mathbb{Q}\right]}-\sum_{\substack{\sqrt{2} \in K_{2 v}, 2 v \\ 2 \nmid v}} \frac{h_{\psi}(v)}{\left[K_{2 v, 2 v}: \mathbb{Q}\right]},
$$

with $h_{\psi}$ the Dirichlet convolution of $\psi$ and the Möbius function, i.e., $h_{\psi}(n)=$ $\sum_{d \mid n} \psi(d) \mu(n / d)$.

Remarkably, despite its arithmetic complexity, $\delta_{g}(3,4 ; a, 4)$ has some easy properties.

Theorem 5 ([M-1]). (GRH). Write $g= \pm g_{0}^{h}$, where $g_{0}$ is positive and not an exact power of a rational number.

1) If $g>0$ and $h$ is even, then $\delta_{g}(2,3 ; 1,3) \leq \delta_{g}(2,3 ; 2,3)$; otherwise $\delta_{g}(2,3 ; 1,3) \geq \delta_{g}(2,3 ; 2,3)$. We have equality iff $\mathbb{Q}\left(\sqrt{g_{0}}\right)=\mathbb{Q}(\sqrt{3})$ and $\nu_{2}(h) \in\{0,2\}$. The same result holds with $\delta_{g}(2,3 ; *, 3)$ replaced by $\delta_{g}(*, 3)$.

2) If $\delta_{g}(3,4 ; 3,4) \neq \delta_{g}(3,4 ; 1,4)$, then $\operatorname{sgn}\left(\delta_{g}(3,4 ; 3,4)-\delta_{g}(3,4 ; 1,4)\right)=\operatorname{sgn}(g)$.

3) If $g \in \mathcal{G}$ and $2 \nmid a$, then $\delta_{g}(3,4 ; a, 4)+\delta_{-g}(3,4 ; a, 4)=1 / 4$.

Let $\delta(p ; a, d)$ denote the density of elements in $\mathbb{F}_{p}^{*}$ having order congruent to $a(\bmod d)$. It is not so difficult to show that the average density $\delta(a, d)$ of elements of order congruent to $a(\bmod d)$ in a field of prime characteristic exists; i.e., we have $\lim _{x \rightarrow \infty} \sum_{p \leq x} \delta(p ; a, d) / \pi(x)=\delta(a, d)$, where $\pi(x)$ denotes the number of primes $p \leq x$. The quantity $\delta(a, d)$ can be studied by fairly elementary methods, but nevertheless turns out to exhibit behaviour similar to $\delta_{g}(a, d)$. An interpretation of $\delta(a, d)$ is that it is the $g$-average of $\delta_{g}(a, d)$ :

Theorem 6 ([M-3]). (GRH). We have

$$
\frac{1}{2 x} \sum_{|g| \leq x} \delta_{g}(a, d)=\delta(a, d)+O\left(\frac{1}{\sqrt{x}}\right)
$$


In some sense, if one takes out the Galois theory and degree aspects of formula (1), one obtains $\delta(a, d)$. More precisely, if one sets $c_{g}(1+t a, d t, n t)=1$ and $\left[K_{[d, n] t, n t}: \mathbb{Q}\right]=\varphi([d, n] t) n t$ (this is the maximal degree possible), then it can be shown that one obtains $\delta(a, d)$ out of $\delta_{g}(a, d)$ ( M-Av $)$. One has $\delta($ odd, 4$)=1 / 6$. It is not difficult to prove that for most integers $g$ with $|g| \leq x$ we have that $D(g)$ has a prime divisor congruent to $1(\bmod 4)$. (Indeed, the size of the exceptional set is bounded above by $\ll_{g} x / \sqrt{\log x}$.) Thus from Theorem 3 we infer that for almost all integers $g$ with $|g| \leq x$ we have, on GRH, $\delta_{g}($ odd, 4$)=\delta($ odd, 4$)=1 / 6$. If $\delta_{g}(a, 4) \neq \delta(a, 4)$, then the difference will be small in absolute value, as is also obvious from Theorem 3. It turns out that these phenomena hold true in general. In case $d$ equals a prime power it is still possible to write down an explicit formula for the density $\delta_{g}(a, d)$ from which the latter two properties can be similarly inferred [M-2]. For general $d$ this seems to be difficult. Nevertheless, the following two results can be proved (where $k(d)=\prod_{p \mid d} p$ is the squarefree kernel of $d$ ):

Theorem 7 (M-3]). (GRH). Let $d$ be fixed. There are at most $O_{d}\left(x \log ^{-1 / \varphi\left(k_{1}(d)\right)} x\right)$ integers $g$ with $|g| \leq x$ such that $\delta_{g}(a, d) \neq \delta(a, d)$ for some integer $a$. In particular,

$$
\left(\delta_{g}(0, d), \ldots, \delta_{g}(d-1, d)\right)=(\delta(0, d), \ldots, \delta(d-1, d))
$$

for almost all integers $g$, where

$$
k_{1}(d)=\left\{\begin{array}{ll}
k(d) & \text { if } d \text { is odd; } \\
4 k(d) & \text { otherwise, }
\end{array} \text { and } k_{2}(d)= \begin{cases}k(d) & \text { if } d \text { is odd } ; \\
(4, d / 2) k(d) & \text { otherwise }\end{cases}\right.
$$

Theorem 8 ([M-3]). (GRH). Suppose that $g \in \mathcal{G}$. Set $D_{1}=|D(g) /(D(g), d)|$. Then

$$
\left|\delta_{g}(a, d)-\delta(a, d)\right|<\frac{3 \cdot 2^{\omega\left(D_{1}\right)+2}}{\varphi\left(D_{1}\right) D_{1}},
$$

where $\omega(n)$ denotes the number of distinct prime divisors of $n$.

The following basic result reduces the study of $\delta_{g}(a, d)$ to that of $\delta_{g}\left(a, k_{2}(d)\right)$.

Theorem 9 ([M-3]). (GRH).

1) If $q$ is an odd prime dividing $d_{1}$, then $\delta_{g}\left(a, q d_{1}\right)=\delta_{g}\left(a, d_{1}\right) / q$.

2) If $8 \mid d_{1}$, then $\delta_{g}\left(a, 2 d_{1}\right)=\delta_{g}\left(a, d_{1}\right) / 2$.

That is, we have $\delta_{g}(a, d)=\delta_{g}\left(a, k_{2}(d)\right) k_{2}(d) / d$.

It is easy to see that $\delta(a, d)$ satisfies a similar and slightly stronger property: $\delta(a, d)=\delta(a, k(d)) k(d) / d$.

In Theorem 4 it is seen that there is a difference in behaviour of $\operatorname{ord}_{p}(g)$ when $p$ is restricted to those primes with $p \equiv 1(\bmod 4)$, respectively $p \equiv 3(\bmod 4)$. A similar phenomenon (having a Galois-theoretic explanation) is seen to hold in general.

Theorem 10 ([M-3]). (GRH). Suppose that $(a, d)=(b, d)=1$.

1) If $d$ is odd, then $\delta_{g}(1, k(d) ; a, d)=\delta_{g}(1, k(d) ; b, d)$.

2) If $d$ is even, then $\delta_{g}(1,2 k(d) ; a, d)=\delta_{g}(1,2 k(d) ; b, d)$.

On the other hand, if $(a, d) \neq(b, d)$, then it seems that rarely $\delta_{g}(a, d)=\delta_{g}(b, d)$; cf. Theorem 3 with the first part of Theorem 4 . 


\section{On the computation of $\delta_{g}(a, d)$}

As in Theorem 3, in general $\delta_{g}(a, d)$ can be expressed in terms of linear combinations of the constants $A_{\chi}$ with coefficients coming from certain cyclotomic fields:

Theorem 11 (畏]). (GRH). Let $a$ and $d$ be arbitrary natural numbers. Then there exists an integer $d_{1} \mid k_{1}(d)$ such that

$$
\delta_{g}(a, d)=\sum_{\chi \in G_{d_{1}}} c_{\chi} A_{\chi} \text { with } c_{\chi} \in \mathbb{Q}\left(\zeta_{o_{\chi}}\right),
$$

where $c_{\chi}$ can be explicitly computed, $G_{d_{1}}$ denotes the group of Dirichlet characters modulo $d_{1}$, and o $o_{\chi}$ is the order of $\chi$ in $G_{d_{1}}$.

The following result allows one to evaluate the constants $A_{\chi}$ easily with ten decimal digit precision and hence, by Theorem 11, the density $\delta_{g}(a, d)$.

Theorem 12 ( $\mathrm{M}-\mathrm{Av})$. Let $p_{1}(=2), p_{2}, \ldots$ be the sequence of consecutive primes. Let $\chi$ be any Dirichlet character and $n \geq 31$ (hence $p_{n} \geq 127$ ). Then

$$
\begin{gathered}
B_{\chi}=A L(2, \chi) L(3, \chi) L(4, \chi) S(n) R_{1}, \\
S(n)=\prod_{k=1}^{n}\left(1+\frac{\chi\left(p_{k}\right)}{p_{k}\left(p_{k}^{2}-p_{k}-1\right)}\right)\left(1-\frac{\chi\left(p_{k}\right)}{p_{k}^{3}}\right)\left(1-\frac{\chi\left(p_{k}\right)}{p_{k}^{4}}\right), \\
B_{\chi}=\prod_{p}\left(1+\frac{[\chi(p)-1] p}{\left[p^{2}-\chi(p)\right](p-1)}\right)=A_{\chi} \prod_{p \mid d}\left(1-\frac{1}{p(p-1)}\right), \\
A=\prod_{p}\left(1-\frac{1}{p(p-1)}\right)=0.3739558136 \cdots, \text { and } \frac{1}{1+p_{n+1}^{-3.85}} \leq\left|R_{1}\right| \leq 1+\frac{1}{p_{n+1}^{3.85} .}
\end{gathered}
$$

The factor $A L(2, \chi) L(3, \chi) L(4, \chi)$ in the latter result is the beginning of an expansion of $B_{\chi}$ in terms of special values of $L$-series:

Theorem 13 ( $\mathrm{M}-\mathrm{Av}])$. One has

$$
B_{\chi}=A \frac{L(2, \chi) L(3, \chi)}{L\left(6, \chi^{2}\right)} \prod_{r=1}^{\infty} \prod_{k=3 r+1}^{\infty} L\left(k, \chi^{r}\right)^{\lambda(k, r)}, \text { with } \lambda(k, r) \in \mathbb{Z} .
$$

This formula can be used to approximate $B_{\chi}$ (and thus $A_{\chi}$ ) with even higher numerical precision. The integers $\lambda(k, r)$ are related to so-called convoluted Fibonacci numbers (see [M-Fi]) and exhibit certain monotonicity properties in both the $k$ and $r$ directions ([M-Fi $)$. These monotonicity properties are valid in a much more general setting; see [M-Wi].

\section{ON SIMILAR RESUltS FOR THE INDEX}

The index, $\left[(\mathbb{Z} / p \mathbb{Z})^{*}:\langle g(\bmod p)\rangle\right]$, of the subgroup generated by $g(\bmod p)$ inside the multiplicative group of residues mod $p$, is denoted by $r_{p}(g)$ and called the (residual) index mod $p$ of $g$. For this quantity, similar questions can be asked with $\operatorname{ord}_{p}(g)$ replaced by $r_{p}(g)$. The results under this replacement turn out to be rather similar (see [M-1, $\mathrm{M}-2, \mathrm{M}-3, \mathrm{P}]$ ); however, they are much easier to establish. A reason for this is that in the latter case, intersection coefficients do not appear. It was in this context that the constants $A_{\chi}$ were introduced by Pappalardi $[\mathrm{P}$. 


\section{ON THE PROOFS OF THE RESULTS}

For reasons of space we can only give a small sample here. We sketch the proof of Theorem 2

Sketch of the proof of Theorem 2. On noting that $r_{p}(g) \operatorname{ord}_{p}(g)=p-1$ we obtain that $N_{g}(a, d)(x)=\sum_{t=1}^{\infty} V_{g}(a, d ; t)(x)$, where

$$
V_{g}(a, d ; t)(x):=\#\left\{p \leq x: r_{p}(g)=t, p \equiv 1+t a(\bmod d t)\right\} .
$$

In this infinite sum the terms with $t \geq \sqrt{\log x}$ are less easily individually computed, but since they are small, they can be taken together to form an error term, which can be estimated by $O\left(x \log ^{-3 / 2} x\right)$. If $(1+t a, d)>1$, then there is at most one prime counted by $V_{g}(a, d ; t)(x)$ and this prime has to divide $d$. In this way one obtains that

$$
N_{g}(a, d)(x)=\sum_{t \leq \sqrt{\log x},(1+t a, d)=1} V_{g}(a, d ; t)(x)+O\left(\frac{x}{\log ^{3 / 2} x}\right) .
$$

Note that $V_{g}(a, d ; 1)(x)$ counts the number of primes $p \equiv 1+a(\bmod d)$ such that, moreover, $g$ is a primitive root modulo $p$. This function, and indeed $V_{g}(a, d ; t)(x)$, can be estimated by a variation of Hooley's classical argument $[\mathrm{H}]$. However, we need to carry this out with a certain uniformity in $t$, which forces us to keep track of the dependence on $t$ of the various estimates. Furthermore, as will be explained shortly, the additional condition $p \equiv 1+t a(\bmod d t)$ is responsible for bringing in the Galois-theoretic intersection coefficients $c_{g}(1+t a, d t, n t)$. By inclusion and exclusion we find that

$$
V_{g}(a, d ; t)(x)=\sum_{n=1}^{\infty} \mu(n) \#\left\{p \leq x: p \equiv 1+t a(\bmod d t), n t \mid r_{g}(p)\right\} .
$$

The counting functions in the latter sum can be estimated by an effective form of Chebotarev's density theorem; cf. Theorem 3 of [M-1] and the discussion immediately following that theorem. Namely, we are interested in those primes $p \equiv 1+a t(\bmod d t)$ that split completely in $K_{n t, n t}:=\mathbb{Q}\left(\zeta_{n t}, g^{1 / n t}\right)$. These primes must have a Frobenius $\sigma$ in $K_{[n, d] t, n t}$ with the property that $\left.\sigma\right|_{\mathbb{Q}\left(\zeta_{d t}\right)}=\sigma_{1+t a}$ and $\left.\sigma\right|_{K_{n t, n t}}=$ id. If such a $\sigma$ exists, then certainly we must have $\left.\sigma_{1+t a}\right|_{\mathbb{Q}\left(\zeta_{d t}\right) \cap K_{n t, n t}}$ $=$ id, i.e., $c_{g}(1+t a, d t, n t)=1$. Indeed, such a $\sigma$ turns out to exist iff $c_{g}(1+t a, d t, n t)$ $=1$. On applying Chebotarev's density theorem one then finds, assuming the Riemann Hypothesis $(\mathrm{RH})$ holds for the field $K_{[d, n] t, n t}$, that

$$
\#\left\{p \leq x: p \equiv 1+t a(\bmod d t), n t \mid r_{g}(p)\right\}=\frac{c_{g}(1+t a, d t, n t)}{\left[K_{[d, n] t, n t}\right]} \operatorname{Li}(x)+O(\sqrt{x} \log x) .
$$

Again there is a problem with the tail in the series in (3), but again it can be reasonably estimated and one obtains that

$$
V_{g}(a, d ; t)(x)=\sum_{P(n) \leq(\log x) / 6} \mu(n) \#\left\{p \leq x: p \equiv 1+t a(\bmod d t), n t \mid r_{g}(p)\right\}+E(x),
$$

where $P(n)$ denotes the greatest prime factor of $n$ and $E(x)$ is the estimate for the tail. On combining the latter two displayed estimates, one then arrives at a usable estimate for $V_{g}(a, d ; t)(x)$. On combining this with (2), the proof of Theorem 2 is then easily completed. 
Working with the sharpest known unconditional version of Chebotarev's density theorem leads to an error term which is too weak for our purposes. Actually, as is clear from the above sketch, it is not required to assume GRH. It suffices to assume $\mathrm{RH}$ for the number fields involved in the proof. So for Theorem 2 it suffices to require $\mathrm{RH}$ for the number fields $K_{[d, n] t, n t}$ with $n$ squarefree, $(n, d) \mid a$ and $(1+t a, d)=1$.

\section{Numerical EXPERIMENTS}

The problem considered here allows for numerical experiments. We give here a small sample of data so obtained. In the cases studied, the numerics seemed to agree well with the theoretical predictions.

TABLe 1. Experimental and theoretical densities for $d=5$

\begin{tabular}{|c|c|c|c|c|c|}
\hline$\delta \backslash a$ & 0 & 1 & 2 & 3 & 4 \\
\hline$\delta(*, 5)$ & $0.20833 \underline{3}$ & $0.23542 \underline{1}$ & $0.17799 \underline{3}$ & $0.23400 \overline{3}$ & $0.14424 \overline{8}$ \\
\hline$\approx \delta_{-11}(*, 5)$ & 0.208347 & 0.235422 & 0.178007 & 0.233974 & 0.144250 \\
\hline$\delta_{-11}(*, 5)$ & $\delta(0,5)$ & $\delta(1,5)$ & $\delta(2,5)$ & $\delta(3,5)$ & $\delta(4,5)$ \\
\hline$\approx \delta_{-5}(*, 5)$ & 0.208348 & 0.264146 & 0.194858 & 0.233282 & 0.099365 \\
\hline$\delta_{-5}(*, 5)$ & $\delta(0,5)$ & $0.26413 \underline{5}$ & $0.19486 \overline{5}$ & $0.23329 \overline{4}$ & $0.09937 \underline{\underline{1}}$ \\
\hline$\approx \delta_{2}(*, 5)$ & 0.208333 & 0.240673 & 0.178706 & 0.229270 & 0.143017 \\
\hline$\delta_{2}(*, 5)$ & $\delta(0,5)$ & $0.24068 \overline{1}$ & $0.17869 \overline{1}$ & $0.22926 \underline{4}$ & $0.14302 \underline{9}$ \\
\hline$\approx \delta_{5}(*, 5)$ & 0.208348 & 0.232581 & 0.292840 & 0.054488 & 0.211742 \\
\hline$\delta_{5}(*, 5)$ & $\delta(0,5)$ & $0.23258 \overline{5}$ & $0.29284 \overline{8}$ & $0.05449 \overline{3}$ & $0.21173 \overline{7}$ \\
\hline
\end{tabular}

If an entry is in a row labelled $\approx \delta_{g}(*, 5)$ and in column $a$, then the number given equals $N_{g}(a, 5)(x) / \pi(x)$ rounded to 6 decimals with $x=2038074743$ (and hence $\left.\pi(x)=10^{8}\right)$. The theoretical values are given with 6 digit precision, with a bar over the last digit indicating that if the number is to be rounded off, it should be rounded upwards. The density $\delta(0,5)=5 / 24$ (unconditional result).

\section{ACKNOWLEDGEMENTS}

Most of my papers mentioned in the references were written whilst I was working in the PIONEER-group of Prof. E. Opdam at the University of Amsterdam (20002004). Several of these papers were completed whilst I was enjoying the inspiring atmosphere of the Max-Planck-Institute in Bonn. I thank both institutes for their hospitality. The data given in Table 1 were calculated using a $C^{++}$program kindly written by Dr. Yves Gallot. My special thanks go to Dr. Paul Tegelaar for his unfailing support and interest over the years.

\section{REFERENCES}

[CM] K. Chinen and L. Murata, On a distribution property of the residual order of $a(\bmod p)$, I, II, J. Number Theory 105 (2004), 60-81, 82-100. MR2032442 (2005c:11117) MR2032443 (2005c:11118)

[H] C. Hooley, On Artin's conjecture, J. Reine Angew. Math. 225 (1967), 209-220. MR0207630 (34:7445)

[M-Av] P. Moree, On the average number of elements in a finite field with order or index in a prescribed residue class, Finite Fields Appl. 10 (2004), 438-463. MR 2067608(2005f:11219) 
[M-Fi] P. Moree, Convoluted convolved Fibonacci numbers, J. Integer Seq. 7 (2004), Article 04.2.2, 16 pp. (electronic). MR 2084694(2005i:11021)

[M-Wi] P. Moree, The formal series Witt transform, Discrete Math. 295 (2005), 143-160. MR2143453 (2006b:05015)

[M-0] P. Moree, On primes $p$ for which $d$ divides $\operatorname{ord}_{p}(g)$, Funct. Approx. Comment. Math. 33 (2005), 85-95.

[M-1] P. Moree, On the distribution of the order and index of $g(\bmod p)$ over residue classes I, J. Number Theory 114 (2005), 238-271. MR2167970(2006e:11152)

[M-2] P. Moree, On the distribution of the order and index of $g(\bmod p)$ over residue classes II, J. Number Theory 117 (2006), 330-354. MR2213769

[M-3] P. Moree, On the distribution of the order and index of $g(\bmod p)$ over residue classes III, arXiv:math.NT/0405527, Journal of Number Theory, to appear.

[P] F. Pappalardi, On Hooley's theorem with weights, Number theory, II (Rome, 1995), Rend. Sem. Mat. Univ. Politec. Torino 53 (1995), 375-388. MR.1452393 (98c:11102)

[W-1] K. Wiertelak, On the density of some sets of primes, IV, Acta Arith. 43 (1984), 177-190. MR0736730 (86e:11081)

[W-2] K. Wiertelak, On the density of some sets of primes $p$, for which $n \mid \operatorname{ord}_{p}(a)$, Funct. Approx. Comment. Math. 28 (2000), 237-241. MR1824009 (2003a:11120)

[Z] D. Zagier, personal communication.

Max-Planck-Institut für Mathematik, Vivatsgasse 7, D-53111 Bonn, Germany

E-mail address: moree@mpim-bonn.mpg.de 